\title{
New Poetry
}

\section{Marcella Polain \\ Boiling jam at Christmas}

This year it's Spring up to Christmas. Daily, Jacarandas burst and I stand surprised along the fence-line between us and the abandoned house:

our plum trees are still burdened, their slim arms bowed by the weight of fruit.

Above, a white screech of cockatoos splits the blue.

At my feet, the long grass rustles.

I tumble scores of purple plums into my skirt, take to others with a spade, walk home slowly, my belly rolling.

Under incandescent light, I prepare the sink, inspect each one, bath them all, pat them dry and lay them on the cool steel bench.

The firm are packed inside the body of our fridge;

the soft and broken set aside.

Over the greased preserving pan, I press two fingers into them, flick out their stony hearts

and make a red flesh heap.

My jam boils fast and thick.

My hands run red, red, red and I clean everything,

set out the jars, snip lengths of silver ribbon,

circles of gold paper.

I do not think of the abandoned house, the rustling fence-line in the failing light or the night-garden, busy with its shallow graves. 